\title{
Proteínas Totales, Fosfatasa Alcalina, Prostaglandinas E2 y Lisozima como Biomarcadores Salivales en Pacientes Adultos con Periodontitis Crónica
}

\author{
Total Protein, Alkaline Phosphatase, Prostaglandin E2 and Lysozyme \\ as Salivary Biomarkers in Patients with Chronic Periodontitis
}

Camila Romero R.; María Suarez M. \& Carmen Gloria Narváez C.

ROMERO, R. C. SUAREZ, M. M. \& NARVÁEZ, C. C. G. Proteínas totales, fosfatasa alcalina, prostaglandinas E2 y lisozima como biomarcadores salivales en pacientes adultos con periodontitis crónica. Int. J. Odontostomat., 11(4):381-385, 2017.

RESUMEN: La periodontitis crónica es una inflamación de los tejidos que rodean y dan soporte a los dientes. Diversos biomarcadores químicos y pro inflamatorios están aumentados durante el transcurso de la enfermedad. El objetivo del estudio fue determinar los distintos niveles salivales de proteínas totales, fosfatasa alcalina, prostaglandina E2 (PGE2) y lisozima en pacientes con periodontitis crónica. Se obtuvieron muestras de saliva de 31 pacientes con periodontitis crónica y se realizó un estudio de serie de casos para la determinación cuantitativa de los biomarcadores. La concentración de proteínas totales se encontró por sobre los rangos de referencia en 22 pacientes, la actividad de la fosfatasa alcalina se vio aumentada en 9 pacientes y la concentración de PGE2 se vio por sobre los rangos de referencia en 30 pacientes. Las proteínas totales y PGE2 son biomarcadores salivales en estos pacientes con periodontitis, no así la fosfatasa alcalina y la lisozima.

PALABRAS CLAVE: proteínas, fosfatasa alcalina, prostaglandina E2, lisozima, biomarcadores salivales.

\section{INTRODUCCIÓN}

La enfermedad periodontal (EP) es una inflamación e infección de los tejidos que rodean y dan soporte a los dientes. Esta presenta como características la acumulación de placa supragingival y subgingival, inflamación gingival, formación de saco periodontal, pérdida de inserción periodontal, pérdida del hueso alveolar y supuración ocasional (Carranza \& Ubios, 2010). En un estudio realizado por Carvajal et al. (2016), a una muestra representativa de mayores de 18 años pertenecientes a Santiago, Chile, se obtuvo que el $80,2 \%$ de ellos presentaron $\geq 50 \%$ de sus sitios periodontales con presencia de sangrado a la exploración lo que se relaciona con la alta prevalencia de enfermedad periodontal en individuos de 35 a 44 años $(90,78 \%)$ y de $100 \%$ en los individuos de 65-74 años (Gamonal et al., 2010).

La periodontitis como infección se relaciona con un grupo de bacterias Gram negativas, Agregatibacter actinomycetencomitans, Porphyromonas gingivalis y
Tannarella forsythia, dentro de las cuales la $P$. gingivalis y $T$. fosythia son responsables de producir periodontitis crónica (Socransky et al., 1998). Aunque estos patógenos son necesarios para producir la enfermedad, no es el único factor necesario para desencadenarla. Se dice que la expresión clínica de la enfermedad se debe a otros factores tales como la respuesta del huésped frente a la virulencia y la magnitud de la carga microbiana. En respuesta, los patógenos periodontales y sus endotoxinas, las células inmunes del periodonto, especialmente los monocitos, secretan biomarcadores pro inflamatorios como prostaglandinas $\mathrm{E}$, Interleucina1 y factor de necrosis tumoral (Carranza \& Ubios; Al Moharib et al., 2014) y biomarcadores químicos, tales como las proteínas totales, fosfatasa alcalina y lisozima (Narváez, 2012).

Dentro de los biomarcadores pro inflamatorios, las prostaglandinas E2 (PEG2) es un metabolito del áci- 
do araquidónico generado por las ciclooxigenasas, responsables del proceso inflamatorio, las que aumentan en sitios periodontales que muestran inflamación y pérdida de inserción. Este biomarcador tiene como función mediar la respuesta inflamatoria manteniendo el foco infeccioso localizado e incrementando el arribo de células defensoras del huésped. Sin embargo, en concentraciones muy aumentadas desencadena una destrucción tisular exagerada (Kumar et al., 2013). Por otro lado, las proteínas totales salivales contribuyen a mantener el volumen del fluido circulante, transportan sustancias relativamente insolubles, actúan en la inactivación de compuestos tóxicos y en la defensa contra agentes invasores. La determinación de éstas es útil para el monitoreo de cambios asociados a diversos estados de la enfermedad. Lisozima salival, proteína bactericida y fungicida, se encarga de hidrolizar los enlaces $\beta 1-4$ del NAM-NAG del péptidoglicano actuando sobre microorganismos Gram positivos como Gram negativos (Narváez) disminuyendo su concentración en la enfermedad periodontal (Surna et al., 2009).

Otra enzima importante corresponde a la fosfatasa alcalina que es una enzima lisosomal la cual va a estar presente en células osteoblásticas, fibroblastos, neutrófilos y bacteria. Posee una elevada correlación entre su aumento en actividad y progreso de la enfermedad periodontal (Carranza \& Ubios). Además esta enzima sigue siendo considerada un biomarcador ideal para enfermedad periodontal ya que tiene un valor predictivo alto cuando es medida en fluido crevicular gingival (Al Moharib et al.).

El objetivo de este estudio es describir los distintos niveles de biomarcadores químicos y pro inflamatorios presentes en la saliva de pacientes adultos con periodontitis crónica: proteínas totales, fosfatasa alcalina, prostaglandinas E2 y lisozima con valor diagnóstico y/o predictivo.

\section{MATERIAL Y MÉTODO}

El diseño de estudio fue serie de casos. Se reclutó a 31 pacientes mediante el método de revisión selectiva de las fichas periodontales de los pacientes con periodontitis crónica de la Clínica Odontológica de la Universidad del Desarrollo, Chile, seleccionándose aquellos que respondieron a los siguientes criterios de inclusión: adultos con periodontitis crónica, ambos géneros, que se atienden en pregrado de la Clínica Odontológica de la Universidad del Desarrollo. Una vez seleccionados los pacientes se procedió a la entrega, lectura y posterior firma del consentimiento informado, previo a validación del comité ético científico de la Facultad de Odontología, en acuerdo con las normas éticas de la declaración de Helsinki del año 2000.

Para la obtención de la muestra de saliva se utilizaron todos los protocolos sanitarios correspondientes, se les entregó un tubo de ensayo para que depositaran la saliva y se guardó a $4{ }^{\circ} \mathrm{C}$. Posteriormente las muestras fueron centrifugadas por 30 segundos, se extrajo el sobrenadante y fueron traspasadas a tubos Eppendorf de 1,5 mL, donde fueron almacenadas a $-4{ }^{\circ} \mathrm{C}$ para su correcta mantención.

La actividad de fosfatasa alcalina se midió utilizando el KIT ALP 405 de WIENER LAB ${ }^{\circledR}$ para lo cual se utilizó la técnica de reactivo único, mezclando $28 \mathrm{~mL}$ de buffer DEA (dietanolamina) 1,0 mol/L, sales de magnesio $(0,5 \mathrm{mmol} / \mathrm{L})$ con $7 \mathrm{~mL}$ de reactivo $p$-nitrofenil fosfato $(p$ $\mathrm{NFF)} 10 \mathrm{mmol} / \mathrm{L}$. Luego en una cubeta mantenida a 37 ${ }^{\circ} \mathrm{C}$ se colocó $1 \mathrm{~mL}$ de reactivo único el cual se pre incubó unos minutos y se agregó $10 \mu \mathrm{L}$ de saliva. Se mezcló inmediatamente y se disparó simultáneamente el cronómetro, se esperó 20 segundos y se leyó la absorbancia inicial a una longitud de onda de $405 \mathrm{~nm}$. Luego se registró la absorbancia después de 1, 2 y 3 min desde la primera lectura. Se determinó la diferencia promedio de absorbancia / $\mathrm{min}$ (DA/min) restando cada una de la anterior y promediando los valores. Se utilizó este promedio para los cálculos.

Para el cálculo de los resultados se utilizó la siguiente ecuación basada en la curva de calibración, Fosfatasa alcalina (UI/l) a 405 nm: DA/min x 6.812.

Para la obtención de resultados para Proteínas totales se utilizó el KIT PROTEINAS TOTALES AA de WIENER LAB ${ }^{\circledR}$. Se marcaron 3 tubos, uno con la letra $B$ (blanco) que contenía 2,0 mL de complejo EDTA/Cu+2 $13 \mathrm{mmol} / \mathrm{L}$ en hidróxido de sodio $875 \mathrm{mmol} / \mathrm{L}$ y alquilaril polieter (AAP) (Reactivo A) otro con la letra S (Standard) el que contenía $20 \mu \mathrm{l}$ de suero patrón y $2,0 \mathrm{~mL}$ de reactivo $\mathrm{A}$ y otro tubo con la letra $\mathrm{D}$ (desconocido) el que contenía $20 \mu \mathrm{L}$ de saliva y $2,0 \mathrm{~mL}$ de reactivo $A$. Cada uno de estos tubos se incubó durante 15 min a 37 ${ }^{\circ} \mathrm{C}$ y se leyó en el espectrofotómetro a 540 llevando a cero con el blanco de reactivo. Luego se realizó el cálculo de los resultados.

Para PGE2 se prepararon placas Petri con una preparación de tampón barbital a $\mathrm{pH} 8,6$, compuesto por 6,4 $\mathrm{g}$ de ácido barbitúrico, $64,5 \mathrm{~mL}$ de $\mathrm{HCl}(0,1 \mathrm{M})$, 
$350 \mathrm{~mL} \mathrm{H}_{2} \mathrm{O}$ bidestilada, $50 \mathrm{~mL} \mathrm{NaOH}(0,1 \mathrm{M}) . \mathrm{Al} \mathrm{H}_{2} \mathrm{O}$ se le adicionó ácido barbitúrico, se revolvió lentamente, agregando $\mathrm{HCl}$ para ajustar $\mathrm{pH}$. Se calentó hasta 60-70 ${ }^{\circ} \mathrm{C}$, se continuó agitando hasta que se disolvió, se dejó hasta que alcanzó temperatura ambiente. Finalmente, se aforó con $\mathrm{H}_{2} \mathrm{O}$ bidestilada a $500 \mathrm{~mL}$. Se preparó un "Stock" de anticuerpos Anti PGE2 (Calbiochem ${ }^{\circledR}$ ) donde se mezcló $500 \mathrm{mLAnti}$-PGE2 con 9,5 mL tampón barbital en $500 \mathrm{~mL}$. Para preparar las placas de agar se pesaron $0,35 \mathrm{~g}$ de agarosa para electroforesis y $0,42 \mathrm{~g}$ de barbital, se mezcló lo anterior en un vaso precipitado de $100 \mathrm{~mL}$ y se adicionó $14 \mathrm{~mL}$ de tampón barbital pH 8,6, se calentó al microondas por $10 \mathrm{~s}$, se agitó con una varilla de vidrio, luego se calentó por periodos no mayores a $5 \mathrm{~s}$ hasta disolver y observar transparencia. Cuando la temperatura fue igual a $56{ }^{\circ} \mathrm{C}$ se le adicionó al vaso precipitado $500 \mu \mathrm{L}$ de "Stock de anticuerpos de PGE2", se agitó y se vertió rápidamente en las placas, se enfrió a temperatura ambiente por 10 min y posteriormente se llevó al refrigerador por 5 min. Finalmente se hicieron las perforaciones para recibir las muestras de saliva en cada placa. Se depositaron las muestras de saliva en las perforaciones de la muestra. Se lavaron las placas con $\mathrm{H}_{2} \mathrm{O}$ destilada para sacar las sales cuatro veces cada vez por un tiempo de unos 40 segundos aproximadamente, luego se tiñeron con la solución de azul de Coomasie por 5 min. Para prepararla tinción de azul de Coomasie se pesaron $5 \mathrm{~g}$ de azul de Coomasie (en polvo), se le adicionaron $450 \mathrm{~mL}$ de ácido acético, $90 \mathrm{~mL}$ de metanol y se aforaron a 1000 con $\mathrm{H}_{2} \mathrm{O}$ destilada. Luego de teñir las placas con azul de Coomasie se lavaron las placas con una solución de lavado que contenían $450 \mathrm{~mL}$ de ácido acético, $90 \mathrm{~mL}$ de metanol y se aforó a 1000 con $\mathrm{H}_{2} \mathrm{O}$ destilada, por un tiempo de $5 \mathrm{~min}$. Finalmente se secaron a $37^{\circ} \mathrm{C}$ por 10 min y se midieron los halos para hacer los posteriores análisis.
Para la determinación de lisozima se prepararon placas con un medio de cultivo selectivo para Mycrococcus lysodeiticus (liofilizado) mediante el método de Bratlid (1977) modificado para soporte de agar, utilizando perforaciones donde se depositaron $20 \mu \mathrm{L}$ de saliva. Se dejó en la estufa entre $25-37^{\circ} \mathrm{C}$ por 24 horas para posteriormente medir los halos de inhibición.

\section{RESULTADOS}

Se evaluaron un total de 31 pacientes con periodontitis crónica. El rango de edad fue de 20 a 87 años. De los pacientes, 23 tenían hábito tabáquico y 10 según su percepción se consideraban con estrés.

Para la concentración de proteínas totales, 2 pacientes presentaron concentraciones bajo los rangos de referencia, 7 pacientes presentaron concentraciones dentro de los rangos de referencia y 22 pacientes presentaron concentraciones por sobre los rangos de referencia. De este último grupo, 16 pacientes correspondían a mujeres y 6 pacientes a hombres, 7 tenían hábito tabáquico y 8 pacientes se consideraban con estrés (Tabla I). La concentración de proteínas totales en 22 pacientes se encontró elevada respecto a los rangos de referencia 3,2 $\pm 1,7$ (UI/L). La actividad enzimática de la fosfatasa alcalina se encuentra dentro de los valores de referencia en 18 casos, por sobre los valores de referencia en 9 casos y bajo estos en 4 casos. De los 9 pacientes donde se presentó un aumento de la actividad, 6 pacientes correspondían a mujeres, 3 pacientes a hombres, 3 pacientes tenían hábito tabáquico y 1 según su percepción se encontraba con estrés (Tabla II). La actividad enzimática se

Tabla I. Concentración de proteínas totales en pacientes con periodontitis crónica en distintos rangos.

\begin{tabular}{lccccc}
\hline$(\mathrm{mg} / \mathrm{mL})$ & $\mathrm{N}$ & Mín. & Máx. & Mediana & Media \pm D.E. \\
\hline $0-1,4$ & 2 & 0,85 & 0,85 & 0,85 & $0,85 \pm 0,00$ \\
$1,5-4,9$ & 7 & 1,70 & 4,25 & 1,70 & $2,34 \pm 1,49$ \\
5 o más & 22 & 5,10 & 77,44 & 11,06 & $14,73 \pm 15,14$ \\
\hline
\end{tabular}

Tabla II. Actividad enzimática de fosfatasa alcalina en pacientes con periodontitis crónica en distintos rangos.

\begin{tabular}{lccccc}
\hline$(\mathrm{UI} / \mathrm{L})$ & $\mathrm{N}$ & Mín. & Máx. & Mediana & Media \pm D.E. \\
\hline $0-16,32$ & 4 & 9,08 & 15,89 & 13,62 & $13,10 \pm 7,93$ \\
$16,33-42,53$ & 18 & 20,44 & 40,87 & 31,79 & $29,52 \pm 8,89$ \\
42,54 o más & 9 & 54,50 & 463,22 & 89,69 & $179,38 \pm 166,27$
\end{tabular}


mantuvo dentro de los rangos de referencia 29,43 \pm 13,10, (Dabra \& Singh, 2012) en 58\% de los pacientes, presentándose un aumento de la actividad en solo un $29 \%$ de los casos.

La actividad enzimática de la PGE2 se encuentra aumentada en casi la totalidad de los pacientes con niveles por sobre los rangos de referencia $7,4 \times$ $10^{-4} \pm 9 \times 10^{-5} \mu \mathrm{g} / \mathrm{mL}$ establecido por Sánchez et al. (2013). Sólo 1 caso dentro de los 31 pacientes quien poseía el valor considerado normal $(0,00073)$, siendo excluido de la tabla de estadígrafos. De estos $30 \mathrm{ca}-$ sos, 22 pacientes correspondían a mujeres, 8 hombres, 8 poseían hábito tabáquico y 10 pacientes se consideraban con estrés (Tabla III).

Respecto de la determinación de la actividad de la lisozima no se evidenció ningún halo de inhibición sobre crecimiento de la bacteria Micrococcus lysodeicus inoculadas en las placas de agar.

Tabla III. Concentración de PGE2 en pacientes con periodontitis crónica en distintos rangos.

\begin{tabular}{cccccc}
\hline$(\mu \mathrm{g} / \mathrm{mL})$ & $\mathrm{N}$ & Mín. & Máx. & Mediana & Media \pm D.E. \\
\hline $0,00074-$ o más & 30 & 0,00135 & 0,00294 & 0,00234 & $0,00231 \pm 0,00038$ \\
\hline
\end{tabular}

\section{DISCUSIÓN}

Teniendo en cuenta las limitaciones de la detección precoz de la enfermedad periodontal crónica, la determinación de biomarcadores efectivos en saliva permitiría tener algún indicador para la detección anticipada de la enfermedad.

La concentración de proteínas totales se encontró elevada respecto a los rangos de referencia, lo que concuerda con resultados previos de Carranza \& Ubios con un mayor aumento de la concentración de proteínas totales en pacientes de género femenino que aquellos de género masculino. Se ha asociado algunos factores en las mujeres que van a influir sobre la expresión de la periodontitis crónica, como la influencia de factores hormonales o el uso de anticonceptivos orales. Se postula que las hormonas ováricas pueden aumentar la inflamación de los tejidos gingivales y exagerar la respuesta de a los irritantes locales, o aquellos pacientes que consumen anticonceptivos orales va a haber una reacción exagerada a irritantes gingivales locales (Carranza \& Ubios).

Se ha descrito un aumento de la actividad de la fosfatasa alcalina en sitios periodontales donde se encuentra fluido gíngivo crevicular (Malhotra et al., 2010), pero en este estudio la actividad enzimática se mantuvo dentro de los rangos de referencia.

La concentración de PGE2 se presentó aumentada en casi la totalidad de las muestras obtenidas. Al encontrarse muy elevada fomenta la destrucción de tejidos blandos y duros, asi como la degradación del colágeno y reabsorción ósea, por lo que también se puede demostrar el avance de la enfermedad periodontal en estos pacientes. El efecto preinflamatorio va a servir para la detección temprana de la enfermedad, pudiendo llegar a un diagnóstico precoz.

Dentro de este estudio se consideró que las proteínas totales y la PGE2 correspondieron a buenos biomarcadores salivales, no así la fosfatasa alcalina, ya que este último como biomarcador es más utilizado para en Fluido Gingival Crevicular.

ROMERO, R. C. SUAREZ, M. M. \& NARVÁEZ, C. C. G. Total protein, alkaline phosphatase, prostaglandin E2 and lysozyme as salivary biomarkers in patients with chronic periodontitis. Int. J. Odontostomat., 11(4):381-385, 2017.

ABSTRACT: Chronic periodontitis is an inflammation of tissue that surrounds and supports the teeth; during the course of the disease there is an increase of different chemical and pro-inflammatory biomarkers. The objective of the study was to determine different levels in saliva of total protein, alkaline phosphatase, lysozyme and prostaglandin E2 (PGE2) in patients with chronic periodontitis. We used saliva samples from 31 patients who had chronic periodontitis and the study was a case of series. Our results showed 22 patients with increased concentrations of protein concentration, nine patients with increased alkaline phosphatase and PGE2 concentration was above the reference range in 30 patients: The total protein and PGE2 are good salivary biomarkers in patients with periodontitis, but not the alkaline phosphatase and lysozyme.

KEY WORDS: proteins, alkaline phosphatase, prostaglandin E2, lysozyme, salivary biomarkers. 
ROMERO, R. C. SUAREZ, M. M. \& NARVÁEZ, C. C. G. Proteínas totales, fosfatasa alcalina, prostaglandinas E2 y lisozima como biomarcadores salivales en pacientes adultos con periodontitis crónica. Int. J. Odontostomat., 11(4):381-385, 2017.

\section{REFERENCIAS BIBLIOGRÁFICAS}

Al Moharib, H. S.; AlMubarak, A.; AlRowis, R.; Geevarghese, A.; Preethanath, R. S. \& Anil, S. Oral Fluid Based Biomarkers in Periodontal Disease: Part 1. Saliva. J. Int. Oral Health, 6(4):95-103, 2014.

Bratlid, D. A simple procedure for determination of bacteriolytic activity in biological fluids. Acta Pathol. Microbiol. Scand. C., 85(1):17-20, 1977.

Carranza, F. \& Ubios, A. Las estructuras de soporte dentario En: Carranza, F. A.; Neuman, M. G.; Takel, H. H.\& Klokkevold, P. R. Periodontología Clínica. Ciudad de México, McGraw-Hill Interamericana, 2010.

Carvajal, P.; Gómez, M.; Gomes, S.; Costa, R.; Toledo, A.; Solanes, F.; Romanelli, H.; Oppermann, R.; Rösing, C. \& Gamonal, J. Prevalence, severity, and risk indicators of gingival inflammation in a multi-center study on South American adults: a cross sectional study. J. Appl. Oral Sci., 24(5):524-34, 2016.

Dabra, S. \& Singh, P. Evaluating the levels of salivary alkaline and acid phosphatase activities as biochemical markers for periodontal disease: A case series. Den. Res. J. (Isfahan), 9(1):41-5, 2012.

Gamonal, J.; Mendoza, C.; Espinoza, I.; Muñoz, A.; Urzúa, I.; Aranda, W.; Carvajal, P. \& Arteaga, O. Clinical attachment loss in Chilean adult population: First Chilean National Dental Examination Survey. J. Periodontol., 81(10):140310, 2010

Kumar, A. K.; Reddy, N. R.; Babu, M.; Kumar, P. M.; Reddy, V. S. \& Chavan, C. V. Estimation of prostaglandin E2 levels in gingival crevicular fluid in periodontal health, disease and after treatment. Contemp. Clin. Dent., 4(3):303-6, 2013.

Malhotra, R.; Grover, V.; Kapoor, A.; Kapur, R. Alkaline phosphatase as a periodontal disease marker. Indian J. Dent. Res., 21(4):531-6, 2010.

Narváez, C. G. Elementos de Bioquímica Para Odontología. Bioquímica Aplicada en Odontología. Concepción; Editorial Academica Española, 2012. pp.78-79,

Sánchez, G. A.; Miozza, V. A.; Delgado, A. \& Buch, L. Salivary IL-1b and PGE2 as biomarkers of periodontal status, before and after periodontal treatment. J. Clin. Periodontol., 40(12):1112-7, 2013.

Socransky, S. S.; Haffajee, A. D.; Cugini, M. A.; Smith, C. \& Kent, R. L. Jr. Microbialcomplexes in subgingival plaque. J. Clin. Periodontol., 25(2):134-44, 1998.

Surna, A.; Kubilius, R.; Sakalauskiene, J.; Vitkauskiene, A.; Jonaitis, J.; Saferis, V. \& Gleiznys, A. Lysozyme and microbiota in relation to gingivitis and periodontitis. Med. Sci. Monit., 15(2):CR66-73, 2009
Dirección para correspondencia:

Carmen Gloria Narváez C.

Facultad de Ciencias de la Salud Universidad del Desarrollo

Concepción

CHILE

E-mail: cgnarvaez@udd.cl

Recibido : 15-04-2015

Aceptado : 24-07-2017 